\title{
A rare case report of primary bone lymphoma and a brief review of the literature
}

This article was published in the following Dove Press journal:

OncoTargets and Therapy

9 August 2016

Number of times this article has been viewed

\author{
Jia Wang ${ }^{1,2}$ \\ Shouren Fan ${ }^{1,3}$ \\ Jie Liư ${ }^{2}$ \\ Bao Song ${ }^{4}$
}

'School of Medicine and Life Sciences, University of Jinan-Shandong Academy of Medical Sciences, ${ }^{2}$ Department of Oncology, Shandong Cancer Hospital affiliated to Shandong University, ${ }^{3}$ Department of Intervention, Qihe People's Hospital, ${ }^{4}$ Elementary Laboratory, Shandong Cancer Hospital affiliated to Shandong University, Jinan, People's Republic of China

Correspondence: Jie Liu

Department of Oncology, Shandong Cancer Hospital affiliated to Shandong University, No 440 Jiyan Road, Huaiyin District, Jinan, Shandong Province 250000, People's Republic of China Email lj691012@।26.com

Bao Song

Elementary Laboratory, Shandong Cancer Hospital affiliated to Shandong University, No 440 Jiyan Road, Huaiyin District, Jinan, Shandong Province 250000,

People's Republic of China

Email songbaojn@126.com

\begin{abstract}
Primary bone lymphoma is a rare and peculiar extranodal presentation of non-Hodgkin's lymphoma, which threatens human health. It can be defined as a lymphoma that occurs in the bone, consisting of a single bone lesion with or without regional lymphadenopathies, and its underlying causes are largely unknown. In this case report, we describe a male who presented with left-sided distal forearm pain, swelling of 2 months duration, and progressive limited wrist motion for about 1 month. The patient had no significant medical history except diabetes. Magnetic Resonance Imaging demonstrated a sheet-like bone destruction area in the left-sided radius, localized discontinuous bone cortex, and adjacent soft tissue masses. Finally, a bone biopsy examined by histopathological and immunochemical methods confirmed a diagnosis of primary bone diffuse large B-cell lymphoma. Due to the rarity of this disease, the level of evidence supporting some diagnostic and therapeutic decisions remains low, and therefore, the details of the rare case may facilitate treatment of similar diseases and provide insight about this obscure lymphoproliferative malignancy. Also, related recent literature reports of primary bone lymphoma are reviewed.

Keywords: DLBCL, diffuse large B-cell lymphoma, primary DLBCL bone lymphoma, radius, consolidation radiotherapy, rituximab
\end{abstract}

\section{Introduction}

Primary bone lymphoma (PBL) is a peculiar extranodal presentation of non-Hodgkin's lymphoma. Primary bone diffuse large B-cell lymphoma (DLBCL) is the most common pathological type, comprising about $80 \%$ of PBL. Diagnosis of PBL depends on the combined clinical examination and imaging studies, and is confirmed with immunohistochemical examination. Due to the rarity of this disease, more relative studies and case reports are needed to provide insight about this obscure lymphoproliferative malignancy. Here, we report one rare case of primary bone DLBCL involving the distal radius. Meanwhile, clinical, imaging, and immunohistochemical aspects, therapeutic options, and related literature review are discussed.

\section{Case presentation}

A 44-year-old man was hospitalized with a history of untreated diabetes, a 2-month history of left-sided distal forearm pain and swelling, and development of progressive limited motion of the left wrist of 1 month duration. At the time of initial presentation, he had left-sided distal forearm pain accompanied with a slight swelling, without fever, weight loss, or fatigue. He had undergone X-ray screening of the left-sided upper extremity in other hospital and the result showed a sheet-like, worm-eaten bone destruction area in the left-sided distal radius. The localized bone cortex was completely or partially discontinuous (Figure 1). After 1 month, with aggravated pain, swelling 


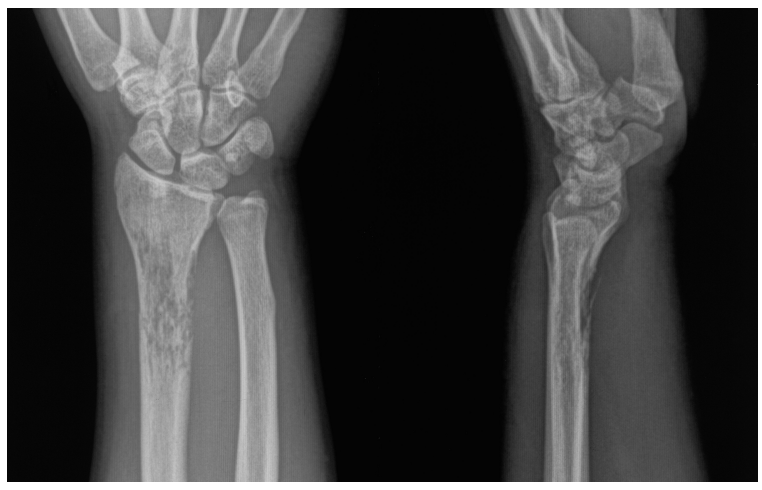

Figure I Sheet-like, worm-eaten bone destruction area is shown in the leftsided distal radius X-ray and localized bone cortex is completely or partially discontinuous.

of left-sided distal forearm, and progressive limited motion in the left wrist, the patient was admitted to our hospital and underwent Magnetic Resonance Imaging, which demonstrated a sheet-like bone destruction area in the left-sided radius, localized discontinuous bone cortex, and adjacent soft tissue masses, hinting at primary bone malignancy (Figure 2). He denied a history of fever of unknown origin and viral infection. Clinically, still the patient had no other symptoms, including fever, weight loss, or fatigue. The physical examination only revealed a slight prominence when palpating the left-sided distal forearm and wrist, without any palpable hepatosplenomegaly or lymphadenopathy. Laboratory tests including the routine viral test were almost normal except blood glucose $(8.1 \mathrm{mmol} / \mathrm{L})$ and alkaline phosphatase (134 U/L). Computed tomography (CT) results of brain, thorax, and abdomen were normal, and the bone marrow aspiration smear did not show neoplastic involvement. A bone biopsy of the lesion and the pathological examinations suggested a diagnosis of primary bone DLBCL. Immunochemical results showed CD20+, CD5+, PAX-5+, MUM-1+, CD3-, CD45RO-, CK-, vimentin-, myogenin-, CD30-, CD10-, Bcl-6-, and Ki67+ (70\%), which demonstrated DLBCL, non-germinal center B-cell-like(non-GCB) (Figure 3). According to the Ann Arbor staging, the patient was I EA stage
A

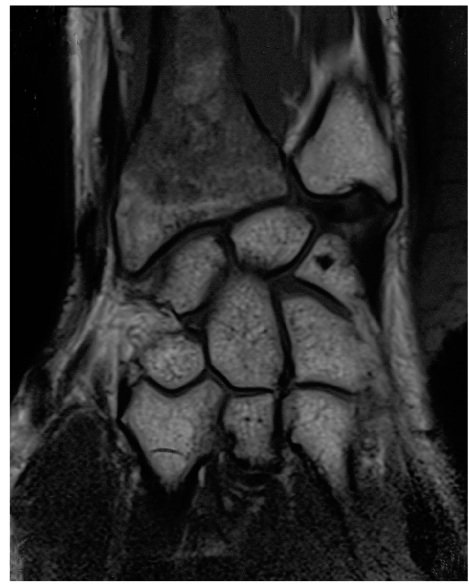

D

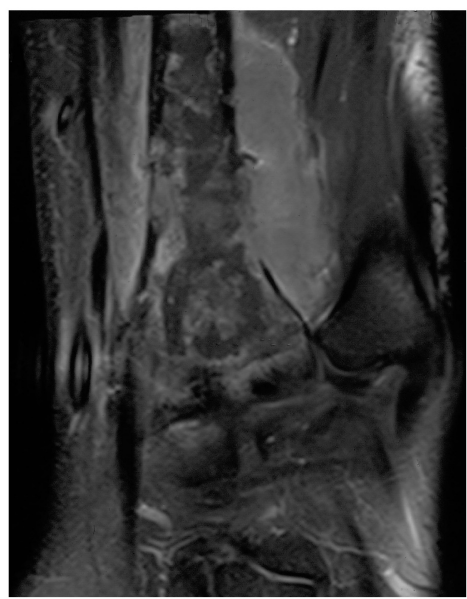

B

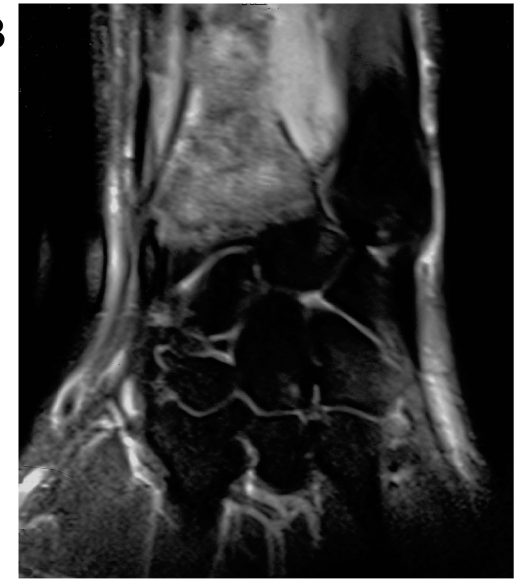

E

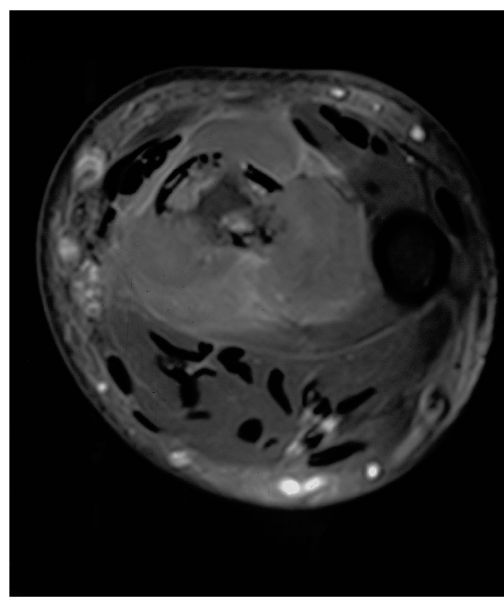

C

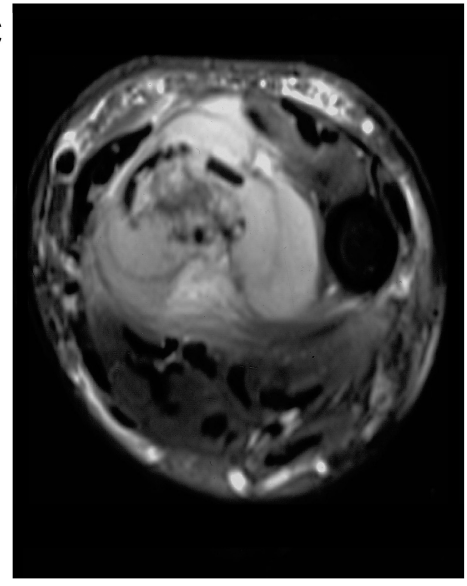

$\mathbf{F}$

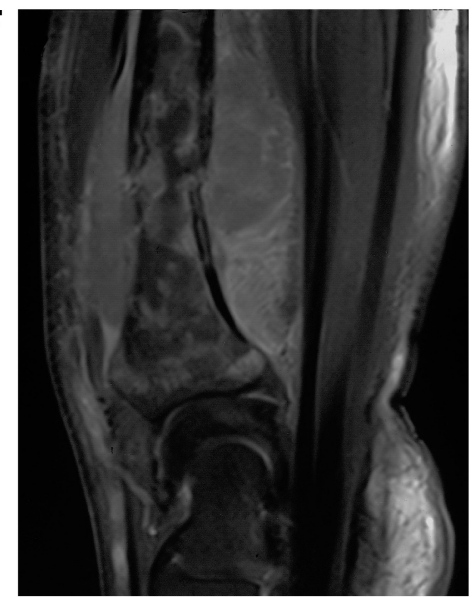

Figure $2 \mathrm{MRI}$ and enhanced MRI: coronary, lateral, sagittal axis.

Notes: MRI shows sheet-like bone destruction area in the left-sided radius, completely or partially discontinuous localized bone cortex, and adjacent soft tissue masses with low-signal intensity on TI-weighted images (A). T2-weighed images show heterogeneous high signal of (B) coronary axis, (C) lateral axis. Enhanced scanning shows heterogeneous enhancement of (D) coronary axis, (E) lateral axis, (F) sagittal axis.

Abbreviation: MRI, magnetic resonance image. 

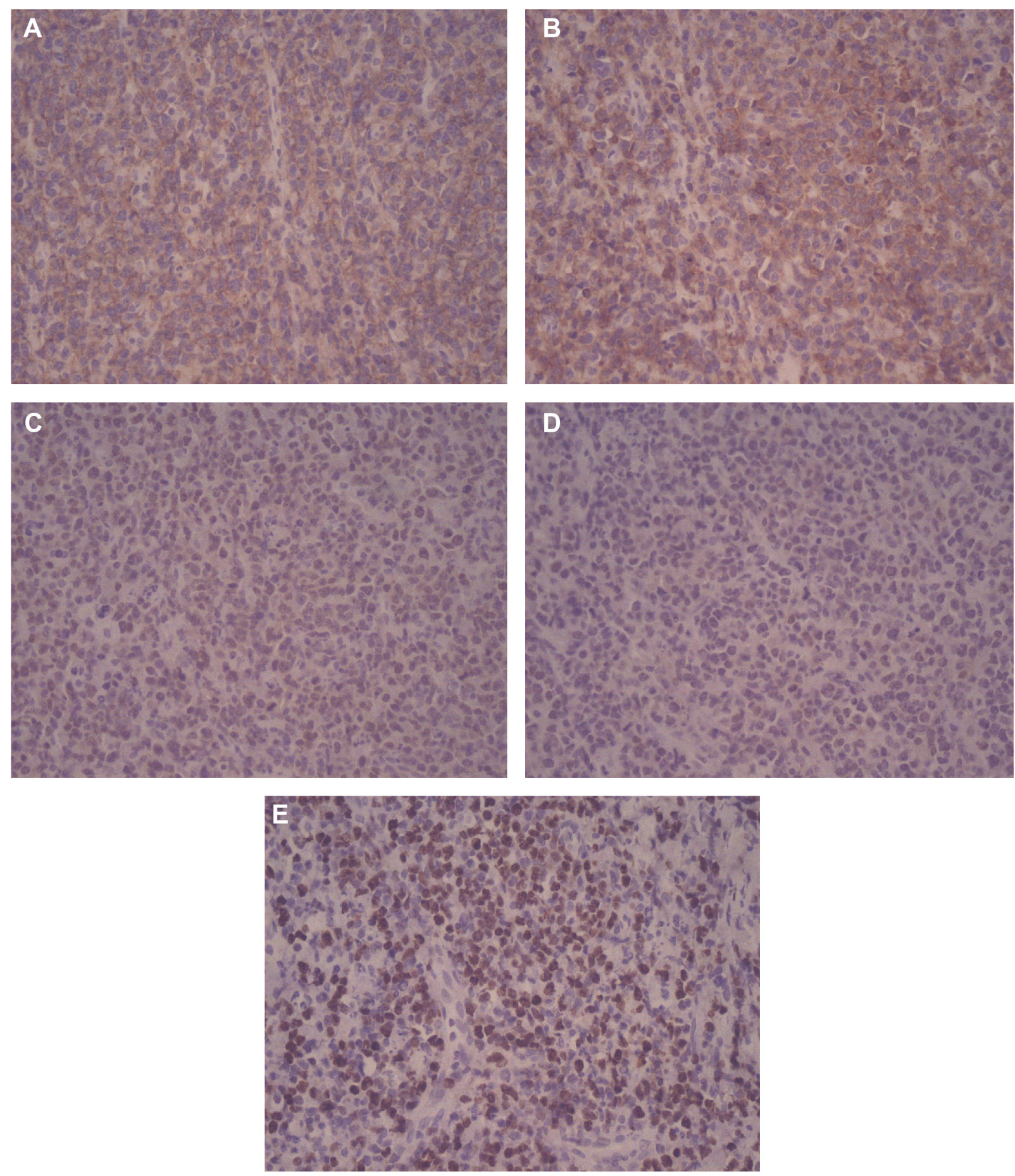

Figure 3 Pathology and immunophenotyping of primary bone diffuse large B-cell lymphoma, non-GCB (×400). (A) CD20+, (B) CD5+, (C) PAX-5+, (D) MUM-I+, (E) Ki67+. Abbreviation: non-GCB, non-germinal center B-cell-like.

of the disease, International Prognostic Index (IPI) score 0. The patient first received four cycles of concurrent chemotherapy with rituximab, cyclophosphamide, doxorubicin, vincristine, and prednisone (R-CHOP). Then he received radiotherapy (RT). The total dose was $32 \mathrm{~Gy} / 16$ fractions. The patient was followed up every 6 months. Up to now, 6 months after treatment, there has been no evidence of recurrence either clinically or radiologically. Informed consent was obtained from the patient for publication of this case report.

\section{Discussion}

According to the World Health Organization classification of tumors of the soft tissue and bone in 2013, ${ }^{1} \mathrm{PBL}$ is defined as a single skeletal lymphoma with or without regional lymph node involvement, and multiple bone lesions without visceral 
or lymph node involvement. Its underlying causes are still largely unknown.

According to rare previous reports, ${ }^{2-7}$ PBL patients generally are characterized by localized bone pain, soft tissue swelling, and possibly a palpable lump in the lesion site. It commonly develops in patients aged between 20 and 50 years, reveals a male preponderance, and the morbidity rate of males is slightly higher than females. ${ }^{8}$ The predilection site of PBL is the femur, accounting for $29 \%$ of all cases, and other sites include the pelvis, humerus, skull and neck, and the tibia. ${ }^{8,9}$ The pooled statistical analysis in the IELSG-14 study shows a low incidence of primary DLBCL bone lymphoma involving the forearm (5.6\%) in PBL patients. ${ }^{9}$

Our patient first presented with distal forearm pain, swelling, and X-ray screening of the left-sided upper extremity showing an osteolytic lesion of his radius. The differential diagnosis included PBL and other primary malignant bone tumors. However, PBL accounts for only $\sim 5 \%$ of the extranodal lymphomas, $<1 \%$ of all non-Hodgkin's lymphomas, and merely about $3 \%-7 \%$ of the malignant bone tumors; ${ }^{8}$ however, all of them could cause pain and swelling in the position of bone lesion. Besides, the radiographic findings of PBL are atypical: bone X-ray usually presents as lytic or osteoblastic lesion, or lesions could even coexist in the same bone; ${ }^{10}$ therefore, it is hard to distinguish the conditions. However, Magnetic Resonance Imaging reveals the extent and details of lesions more clearly than $\mathrm{CT}$ with respect to cubital bones and joints, though the standard contrast-enhanced CT scan is the primary modality for staging, restaging, and follow-up of lymphoma patients.

18FDG positron emission tomography/computed tomography(PET/CT) may have a higher sensitivity and specificity than standard CT scan and was recommended by Lugano Classification as a standard tool for the initial evaluation, staging, and response assessment of FDGavid lymphomas; however, it is only supported by small retrospective studies in lymphoma patients ${ }^{11}$ and has not been confirmed in PBL.

The clinical manifestations and imaging are nonspecific with limitations to distinguish PBL from other primary bone tumors such as Ewing's sarcoma, osteogenic sarcoma, and chondrosarcoma. Therefore, diagnostic samples obtained by surgical procedure and immunohistochemical tests are indispensable for clinicoradiological confirmation of PBL and for differential diagnosis. Other surgical procedures, especially excision of lesions, should be avoided whenever possible, unless the involved weight-bearing bones are destroyed extensively or pathological fracture is present in bone lesions. ${ }^{12,13}$
DLBCL is the most common pathological type either primarily or secondarily infiltrating the skeleton, accounting for about $70 \%-80 \%$ of all $\mathrm{PBL},{ }^{8}$ with rare to anecdotal occurrences of follicular, marginal zone, lymphoplasmacytic, anaplastic large cell, natural killer/T-cell, Burkitt, and Hodgkin lymphomas. Tumor cells are generally immunoreactive for the B-cell markers CD20, CD21, CD45, and CD79a. ${ }^{14,15}$

With the progression of treatment strategies including rituximab and RT which are widely applied in primary bone DLBCL, discrepancies now mostly focus on immunotherapy and RT in rituximab era. Rituximab has essentially changed the natural behavior of DLBCL, especially the primary extranodal disease. A large retrospective study of localized DLBCL and the MINT trial both suggested that patients with primary extranodal disease benefited from the addition of rituximab, ${ }^{16,17}$ while rituximab failed to improve the outcome of patients with DLBCL with skeletal involvement. ${ }^{18}$ Also, the survival benefit of the addition of rituximab to $\mathrm{CHOP}$ in primary bone DLBCL is still controversial. A retrospective British Columbia Cancer Agency study of primary bone DLBCL reported significant improvement in 3-year progression-free survival (PFS) for patients treated with rituximab compared to those who were not ( $88 \%$ vs $52 \%$, respectively). ${ }^{19}$ However, it was questioned by a recent retrospective trial by Tao et al, which insisted that rituximab did not improve outcomes for patients with primary bone DLBCL and speculated that perhaps skeletal DLBCL is a biologic subtype with some resistance to rituximab. ${ }^{20}$

The role of consolidation RT has also been the subject of much debate over the past decades as more trials have emerged that can address the role of RT in the rituximab era. Whether the addition of consolidation RT can improve outcome is still disputable according to a recent retrospective trial series of DLBCL and bulky residual masses. . $^{17,18,21}$ In the prospective RICOVER-60 study for elderly aggressive B-cell lymphoma patients, PFS and overall survival (OS) were found to be inferior without additive RT. ${ }^{22}$ Regarding primary bone DLBCL, the IELSG-14 study also reported that the addition of consolidative post-chemotherapy RT does not further improve the outcome, with both chemoradiotherapy and chemotherapy alone producing a 5-year PFS of $74 \%$ and $67 \%$, respectively. ${ }^{9}$ The British Columbia Cancer Agency study suggested that patients who received RT had significantly worse 10 -year OS than those who received chemotherapy alone ( $25 \%$ vs $56 \%, P=0.025$ ), and the researchers stated that they could not reach conclusions about the role of RT. ${ }^{19}$ On the contrary, the retrospective Rare Cancer Network study suggested anthracycline-based chemotherapy by involved-field RT as the first-line treatment 
for patients with primary bone DLBCL and one of the favorable prognostic factors for OS and lymphoma specific survival was an RT dose $>40$ Gy $(P=0.005) .{ }^{23}$ Randa et al reported that receiving $\mathrm{RT}$ was associated with improved 5-year PFS and had a trend toward an OS benefit, while no significant difference in PFS or OS was found between patients treated with 30-35 Gy versus 36 Gy. ${ }^{20}$ In the IELSG-14 study, there was no significant survival difference between the doses $<36$ Gy and $>36$ Gy in primary bone DLBCL patients. ${ }^{9}$ Compared with previous standard doses of 40-45 Gy, a radiation of 30 Gy is not associated with loss of efficacy in aggressive lymphoma and with no significant differences in in-field relapse rate, PFS and OS.

Although the roles of consolidation $\mathrm{RT}$ and rituximab are still in dispute, our patient was benefited and has not relapsed until now. In early-stage disease, relapses occur equally within and outside the primary sites of disease, with local and systemic relapse rates being $10 \%$ and $17 \%$, respectively. ${ }^{23}$ Many unconfirmed prognostic factors have been reported in earlier retrospective studies. ${ }^{9,19,20,23}$ Conventional IPI plays a prognostic role in patients with advanced-stage DLBCL with skeleton involvement, but not in polyostotic lymphoma and PBLs. ${ }^{9}$ Age, performance status, and serum lactate dehydrogenase levels, the three remaining IPI variables, ${ }^{9}$ and other factors could be used to evaluate the prognosis.

\section{Acknowledgments}

This work was supported by Shandong provincial natural science foundation (ZR2013HM079 and ZR2011HQ021).

\section{Disclosure}

The authors report no conflicts of interest in this work.

\section{References}

1. Rosenberg AE. WHO classification of soft tissue and bone, fourth edition: summary and commentary. Curr Opin Oncol. 2013;25(5):571-573.

2. Marina V, Milena R, Vesna P, Sladana P, Vera A. Complementary roles of bone scintigraphy and MR imaging in the detection and long-term follow-up of primary non-Hodgkin's bone lymphoma in a child-case report. Skeletal Radiol. 2015;44(6):863-868.

3. Mandal PK, Baul S, Dolai TK. Primary bone lymphoma with multifocal osteolytic lesions: a rare case report with review of literature. Blood Res. 2015;50(4):256-260.

4. Inklab M, Steingart RH, Freeman JK. Primary lymphoma of bone presenting as spindle cell neoplasm of the vertebral body: a case report and review of the literature. Case Rep Hematol. 2015;2015:518307.

5. Zhou HY, Gao F, Bu B, et al. Primary bone lymphoma: A case report and review of the literature. Oncol Lett. 2014;8(4):1551-1556.

6. Liu M, Liu B, Han F, Song Y. Primary bone lymphoma of the left radius: a case report and related literature review. Eur J Med Res. 2014; 19(1): 19 .
7. Kishan Prasad HL, Jayaprakash Shetty K, Mathias L, Sunil Kumar Y, Permi HS, Rao C. Primary bone lymphoma of humerus diagnosed by FNAC - a rare case report. Indian J Surg Oncol. 2013;4(3):316-319.

8. Messina C, Christie D, Zucca E, Gospodarowicz M, Ferreri AJ. Primary and secondary bone lymphomas. Cancer Treat Rev. 2015; 41(3):235-246.

9. Bruno Ventre M, Ferreri AJ, Gospodarowicz M, et al. Clinical features, management, and prognosis of an international series of 161 patients with limited-stage diffuse large B-cell lymphoma of the bone (the IELSG-14 study). Oncologist. 2014;19(3):291-298.

10. Shoji H, Miller TR. Primary reticulum cell sarcoma of bone. Significance of clinical features upon the prognosis. Cancer. 1971;28(5): 1234-1244.

11. Cheson BD, Fisher RI, Barrington SF, et al. Recommendations for initial evaluation, staging, and response assessment of Hodgkin and non-Hodgkin lymphoma: the Lugano classification. J Clin Oncol. 2014; 32(27):3059-3068.

12. Liu YC, Gau JP, Yu YB, et al. Prognostic factors and treatment efficacy in patients with primary diffuse large B-cell lymphoma of the bone: single institute experience over 11 years. Intern Med. 2014;53(2): 95-101.

13. Cirakli A, Elli M, Dabak N, Canbaz Tosun F, Dagdemir A, Cirakli S. Evaluation of primary bone lymphoma and the importance of positron emission tomography. Acta Orthop Traumatol Turc. 2014;48(3): 371-378.

14. Pettit CK, Zukerberg LR, Gray MH, et al. Primary lymphoma of bone. A B-cell neoplasm with a high frequency of multilobated cells. Am J Surg Pathol. 1990;14(4):329-334.

15. Huebner-Chan D, Fernandes B, Yang G, Lim MS. An immunophenotypic and molecular study of primary large B-cell lymphoma of bone. Mod Pathol. 2001;14(10):1000-1007.

16. Rieger M, Osterborg A, Pettengell R, et al. Primary mediastinal B-cell lymphoma treated with CHOP-like chemotherapy with or without rituximab: results of the Mabthera International Trial Group study. Ann Oncol. 2011;22(3):664-670.

17. Mian M, Marcheselli L, Rossi A, et al. A diachronic-comparative analysis for the identification of the most powerful prognostic index for localized diffuse large B-cell lymphoma. Ann Oncol. 2014;25(12): 2398-2404.

18. Held G, Zeynalova S, Murawski N, et al. Impact of rituximab and radiotherapy on outcome of patients with aggressive B-cell lymphoma and skeletal involvement. J Clin Oncol. 2013;31(32):4115-4122.

19. Ramadan KM, Shenkier T, Sehn LH, Gascoyne RD, Connors JM. A clinicopathological retrospective study of 131 patients with primary bone lymphoma: a population-based study of successively treated cohorts from the British Columbia Cancer Agency. Ann Oncol. 2007; 18(1):129-135.

20. Tao R, Allen PK, Rodriguez A, et al. Benefit of consolidative radiation therapy for primary bone diffuse large B-cell lymphoma. Int J Radiat Oncol Biol Phys. 2015;92(1):122-129.

21. Phan J, Mazloom A, Medeiros LJ, et al. Benefit of consolidative radiation therapy in patients with diffuse large B-cell lymphoma treated with R-CHOP chemotherapy. J Clin Oncol. 2010;28(27):4170-4176.

22. Held G, Murawski N, Ziepert M, et al. Role of radiotherapy to bulky disease in elderly patients with aggressive B-cell lymphoma. J Clin Oncol. 2014;32(11):1112-1118.

23. Cai L, Stauder MC, Zhang YJ, et al. Early-stage primary bone lymphoma: a retrospective, multicenter Rare Cancer Network (RCN) Study. Int J Radiat Oncol Biol Phys. 2012;83(1):284-291.

24. Lowry L, Smith P, Qian W, et al. Reduced dose radiotherapy for local control in non-Hodgkin lymphoma: a randomised phase III trial. Radiother Oncol. 2011;100(1):86-92. 


\section{Publish your work in this journal}

OncoTargets and Therapy is an international, peer-reviewed, open access journal focusing on the pathological basis of all cancers, potential targets for therapy and treatment protocols employed to improve the management of cancer patients. The journal also focuses on the impact of management programs and new therapeutic agents and protocols on

patient perspectives such as quality of life, adherence and satisfaction. The manuscript management system is completely online and includes a very quick and fair peer-review system, which is all easy to use. Visit http://www.dovepress.com/testimonials.php to read real quotes from published authors.

Submit your manuscript here: http://www.dovepress.com/oncotargets-and-therapy-journal 